\title{
Status of isospin splittings in mesons and baryons
}

\author{
Marek Karliner $\circledast^{1, *}$ and Jonathan L. Rosner $\circledast^{2, ;}$ \\ ${ }^{1}$ School of Physics and Astronomy, Raymond and Beverly Sackler Faculty of Exact Sciences, \\ Tel Aviv University, Tel Aviv 69978, Israel \\ ${ }^{2}$ Enrico Fermi Institute and Department of Physics University of Chicago, \\ 5620 South Ellis Avenue, Chicago, Illinois 60637, USA
}

(Received 23 July 2019; published 15 October 2019)

\begin{abstract}
Current measurements of isospin splittings in mesons and baryons are sufficiently precise that they allow estimates of the mass difference between constituent up and down quarks. Some previous results are updated in the light of these new measurements, and the importance of better measurements of some observables such as $M\left(K^{* \pm}\right), M\left(B^{* 0}\right)-M\left(B^{0}\right)$, and isospin splittings in bottom baryons is noted.
\end{abstract}

DOI: 10.1103/PhysRevD.100.073006

\section{INTRODUCTION}

Isospin-violating mass differences among hadrons are treated in the quark model as a combination of effects. The $u$ and $d$ quarks have an intrinsic mass difference, expressed as a direct contribution to hadron masses and via differing kinetic energies in bound states. Coulomb interactions between quarks depend on the product of their charges times the expectation value of the inverse of their separation. Strong hyperfine interactions between quarks depend on the inverse product of their masses, and electromagnetic hyperfine interactions depend both on that inverse product and on the product of quark charges. One can then write meson and baryon isospin-violating mass differences in terms of a few parameters, yielding sum rules for masses in the limit of small values of these parameters. These were exploited, for example, for mesons with heavy quarks in Ref. [1] and for baryons in Ref. [2]. Isospin splittings in baryons with two heavy quarks were examined in Refs. [3] and [4].

The experimental status of isospin splittings continues to improve. There has been a relatively new measurement of $M\left(D^{*+}\right)-M\left(D^{+}\right)$[5]. Information on masses of individual charge states of charmed and bottom hadrons continues to grow, with exceptional progress in the past year for $\Xi^{c}, \Sigma_{b}$, and $\Xi_{b}$ states [6] (compare PDGLive with the 2018 print version). An update of Ref. [1] was performed about 10 years ago [7]. Even the light-quark sector has seen improvements since the analysis of Ref. [2], driven by the improved

\footnotetext{
*marek@proton.tau.ac.il

†rosner@hep.uchicago.edu
}

Published by the American Physical Society under the terms of the Creative Commons Attribution 4.0 International license. Further distribution of this work must maintain attribution to the author(s) and the published article's title, journal citation, and DOI. Funded by SCOAP. precision in the $\Xi^{0}$ mass measured by the NA48 Collaboration at CERN [8]. An analysis of the present status of isospin splittings in hadrons thus seems appropriate.

We set forth our assumptions, including the interpretation of quarks as constituents with masses of several hundred $\mathrm{MeV}$, in Sec. II. In Sec. III we update analyses of light-quark mesons and baryons. We treat charmed hadrons in Sec. IV, beauty hadrons in Sec. V, and the relation between the two heavy sectors in Sec. VI. We compare our results with those of several other approaches in Sec. VII and conclude in Sec. VIII.

\section{ASSUMPTIONS}

In a constituent-quark framework, hadron masses are governed by the sum of their quark masses, the hyperfine interactions among those quarks, and-for hadrons with more than one heavy quark ( $c$ or $b$ ) —an additional binding term between heavy quarks. This approach $[9,10]$ successfully describes the masses of light-quark hadrons [11], those with a single charm or bottom quark [12], and the mass of the recently observed baryon with two charmed quarks [13].

When the masses of mesons and baryons are fitted with constituent-quark masses and hyperfine interactions, the quark masses in baryons are about $55 \mathrm{MeV}$ heavier than those in mesons [10]. This scheme was used in Ref. [12] to predict $M\left(\Xi_{c c}\right)=(3627 \pm 12) \mathrm{MeV}$, in satisfactory agreement with the observed value [13] $M\left(\Xi_{c c}\right)=(3621.40 \pm$ $0.78) \mathrm{MeV}$. An alternative scheme explains the mass difference by adding a "string-junction" term of $165 \mathrm{MeV}$, allowing one to fit mesons and baryons with a universal set of quark masses [14]. However, this scheme predicts $M\left(\Xi_{c c}\right)$ about $40 \mathrm{MeV}$ higher, so for definiteness we shall stay with the picture of separate quark masses for mesons and baryons. 
TABLE I. Results (in MeV) of a fit to mesons and baryons with additive quark masses (different for mesons and baryons), hyperfine terms, and a binding term $B(s s)$. The label $\bar{m}$ denotes an average between $m_{u}$ and $m_{d}$. The masses of $\phi, \Xi$, and $\Omega$ are corrected by terms $-2 B(s s),-B(s s)$, and $-3 B(s s)$, respectively. We find $\bar{m}^{m}=307.5 \pm 0.33 \mathrm{MeV}, \quad m_{s}^{m}=487.6 \pm 0.51 \mathrm{MeV}, \quad \bar{m}^{b}=362.1 \pm 0.21 \mathrm{MeV}, \quad m_{s}^{b}=543.9 \pm 0.45 \mathrm{MeV}$, $b^{m} /\left(\bar{m}^{m}\right)^{2}=79.4 \pm 0.14 \mathrm{MeV}, \quad b^{b} /\left(\bar{m}^{b}\right)^{2}=50.0 \pm 0.16 \mathrm{MeV}, \quad B(s s)=9.23 \pm 0.50 \mathrm{MeV}$. Errors on each parameter are computed by fixing its value, minimizing $\chi^{2}$ with respect to the other six parameters, and determining what values of the given parameter lead to an increase of $\chi^{2}$ by one unit. The root-mean-square error of the fit is $\sqrt{\sum\left(\Delta M^{2}\right) / 13}=3.85 \mathrm{MeV}$.

\begin{tabular}{|c|c|c|c|c|c|c|c|c|}
\hline Meson & & $\pi$ & \multicolumn{2}{|c|}{$\rho$} & $K$ & \multicolumn{2}{|c|}{$K^{*}$} & $\phi$ \\
\hline Predicted & & 138.5 & \multicolumn{2}{|c|}{773.9} & 494.6 & \multicolumn{2}{|c|}{895.3} & 1019.9 \\
\hline Experiment & & 138.0 & \multirow{2}{*}{\multicolumn{2}{|c|}{$\begin{array}{r}775.2 \\
1.8\end{array}$}} & 495.6 & \multicolumn{2}{|c|}{894.1} & 1019.5 \\
\hline$\Delta M^{2}$ & & 0.2 & & & 1.0 & \multicolumn{2}{|c|}{1.4} & 0.2 \\
\hline Baryon & $N$ & $\Delta$ & $\Lambda$ & $\Sigma$ & $\Sigma^{*}$ & $\Xi$ & $\Xi^{*}$ & $\Omega$ \\
\hline Pred. & 936.5 & 1236.4 & 1118.2 & 1185.0 & 1385.0 & 1329.7 & 1529.4 & 1670.4 \\
\hline Expt. & 938.9 & 1232. & 1115.7 & 1193.2 & 1384.2 & 1321.0 & 1532.5 & 1672.5 \\
\hline$\Delta M^{2}$ & 6.0 & 18.9 & 6.2 & 67.3 & 0.2 & 75.3 & 9.9 & 4.5 \\
\hline
\end{tabular}

Quark masses in [12], from Ref. [11], did not include a small binding term for a pair of $s$ quarks, which we now take into account. The results are shown in Table I. Here the strong hyperfine term is parametrized as

$$
\Delta E_{i j, \mathrm{HFs}}=b\left\langle\sigma_{i} \cdot \sigma_{j}\right\rangle /\left(m_{i} m_{j}\right) .
$$

Superscripts $m$ and $b$ will refer to values in mesons and baryons, respectively. The quark masses differ only slightly from those in Ref. [12].

The model we employ takes into account the intrinsic difference $\Delta=\left(1-\frac{K}{m}\right)\left(m_{u}-m_{d}\right)$ between $u$ and $d$ quarks, where $K$ is a one-body kinetic energy term [15]; Coulomb interactions

$$
\Delta E_{i j \mathrm{em}}=\alpha Q_{i} Q_{j}\left\langle 1 / r_{i j}\right\rangle
$$

between quarks; strong hyperfine (HF) interactions $\Delta E_{i j \mathrm{HFs}}$ as mentioned above; and electromagnetic HF interactions

$$
\Delta E_{i j \mathrm{HFe}}=-\frac{2 \pi \alpha Q_{i} Q_{j}\left|\Psi_{i j}(0)\right|^{2}\left\langle\sigma_{i} \cdot \sigma_{j}\right\rangle}{3 m_{i} m_{j}}
$$

Symbols are defined in Ref. [2]. We may thus write the total isospin splitting as

$$
\begin{aligned}
\sum_{i<j} \Delta E_{i j}= & \langle\Delta\rangle+a \sum_{i<j}\left\langle Q_{i} Q_{j}\right\rangle+b \sum_{i<j}\left\langle\sigma_{i} \cdot \sigma_{j} /\left(m_{i} m_{j}\right)\right\rangle \\
& +c \sum_{i<j}\left\langle Q_{i} Q_{j} \sigma_{i} \cdot \sigma_{j} /\left(m_{i} m_{j}\right)\right\rangle .
\end{aligned}
$$

Separate parameters, labeled by superscripts $m$, will be used for mesons. Henceforth parameters without superscripts will refer to quantities for baryons.

\section{LIGHT-QUARK HADRONS}

\section{A. Mesons}

The isospin splittings of light-quark mesons, based on masses quoted in Ref. [6], are summarized in Table II. Labels denote the change in isospin associated with each mass splitting. The conflict between the quoted $K^{*}$ mass splitting (which we use) and the individual $K^{*}$ masses needs to be resolved before we can take our analysis as definitive.

Each splitting can be written as the sum of terms depending on the parameters $\Delta^{m}, a^{m}, b^{m}$, and $c^{m}$ :

$$
\begin{gathered}
\pi_{2}=\frac{1}{2} a^{m}+\frac{3}{2} \frac{b^{m}}{\left(\bar{m}^{m}\right)^{2}}\left(\frac{\Delta^{m}}{\bar{m}^{m}}\right)^{2}-\frac{3}{2} \frac{c^{m}}{\left(\bar{m}^{m}\right)^{2}}, \\
\rho_{2}=\frac{1}{2} a^{m}-\frac{1}{2} \frac{b^{m}}{\left(\bar{m}^{m}\right)^{2}}\left(\frac{\Delta^{m}}{\bar{m}^{m}}\right)^{2}+\frac{1}{2} \frac{c^{m}}{\left(\bar{m}^{m}\right)^{2}}, \\
K_{1}=\Delta^{m}+\frac{1}{3} a^{m}+\frac{3 b^{m}}{\left(\bar{m}^{m}\right)^{2}} \frac{\Delta^{m}}{m_{s}^{m}}-\frac{c^{m}}{\left(\bar{m}^{m}\right)^{2}} \frac{\bar{m}^{m}}{m_{s}^{m}}, \\
K_{1}^{*}=\Delta^{m}+\frac{1}{3} a^{m}-\frac{b^{m}}{\left(\bar{m}^{m}\right)^{2}} \frac{\Delta^{m}}{m_{s}^{m}}+\frac{c^{m}}{3\left(\bar{m}^{m}\right)^{2}} \frac{\bar{m}^{m}}{m_{s}^{m}} .
\end{gathered}
$$

TABLE II. Isospin splittings of light-quark mesons [6].

\begin{tabular}{lcc}
\hline \hline$\Delta M$ & Label & Value $(\mathrm{MeV})$ \\
\hline$\pi^{ \pm}-\pi^{0}$ & $\pi_{2}$ & $4.5936 \pm 0.0005$ \\
$\rho^{ \pm}-\rho^{0}$ & $\rho_{2}$ & $0.15 \pm 0.42$ \\
$K^{+}-K^{0}$ & $K_{1}$ & $-3.934 \pm 0.020$ \\
$K^{*+}-K^{* 0}$ & $K_{1}^{*}$ & $-6.7 \pm 1.2^{\mathrm{a}}$ \\
\hline \hline
\end{tabular}

\footnotetext{
${ }^{a}$ As quoted in pdgLive. Individual masses quoted are $891.76 \pm$ $0.25 \mathrm{MeV}$ (charged, hadroproduced); $895.5 \pm 0.8 \mathrm{MeV}$ (charged, $\tau$ decay); and $895.55 \pm 0.20 \mathrm{MeV}$ (neutral).
} 
Here we have substituted $\Delta^{m}$ for $m_{u}^{m}-m_{d}^{m}$ in the terms for hyperfine splittings. We then see that four observables are expressed in terms of the three unknowns $\Delta^{m}, a^{m}$, and $c^{m}$. [We use the value of $b^{m} /\left(\bar{m}^{m}\right)^{2}=79.4 \mathrm{MeV}$ from the fit in Table I.] A fit to the observables yields the values $\pi_{2}=4.59 \mathrm{MeV}, \quad \rho_{2}=-0.07 \mathrm{MeV}, \quad K_{1}=-3.93 \mathrm{MeV}$, and $K_{1}^{*}=-3.20 \mathrm{MeV}$, with $\Delta^{m}=-4.12 \pm 0.05 \mathrm{MeV}$, $a^{m}=2.20 \pm 0.62 \mathrm{MeV}, \quad$ and $\quad c^{m} /\left(\bar{m}^{m}\right)^{2}=-2.32 \pm$ $0.21 \mathrm{MeV}$. Here and subsequently the errors on each parameter are obtained by fixing its value, minimizing $\chi^{2}$ with respect to the other two, and determining what values of the given parameter lead to an increase in $\chi^{2}$ by one unit. The $\chi^{2}$ for this fit is 8.77 , nearly all (8.51) contributed by $K_{1}^{*}$. In view of the spread in the Particle Data Group's values for $M\left(K^{* \pm}\right)$ [6], we urge further study of this state.

\section{B. Baryons}

We express the observed mass splittings among the octet baryons and the $\Sigma^{*}$ and $\Xi^{*}$ resonances [6], labeled with subscripts denoting their $\Delta I$ values, summarized in Table III, as functions of $\Delta(u-d$ mass difference with effect on kinetic energies), $a$ (Coulomb interaction), $b$ (strong HF interaction), and $c$ (electromagnetic HF interaction). We have neglected effects of two-body kinetic energy operators and additional small corrections [15]. This decomposition is summarized below, where we have linearized expressions from Ref. [4] in $\Delta$ and where $\bar{m}$ is the average of $m_{u}$ and $m_{d}$ :

$$
\begin{gathered}
N_{1}=\Delta+\frac{a}{3}-\frac{2 b \Delta}{\bar{m}^{3}}+\frac{c}{3 \bar{m}^{2}}, \\
\Sigma_{1}=2 \Delta-\frac{a}{3}+\frac{2 b \Delta}{\bar{m}^{3}}\left[-1+2 \frac{\bar{m}}{m_{s}}\right]+\frac{c}{3 \bar{m}^{2}}\left[1+4 \frac{\bar{m}}{m_{s}}\right] \\
=N_{1}+\Xi_{1}, \\
\Sigma_{1}^{*}=2 \Delta-\frac{a}{3}-\frac{2 b \Delta}{\bar{m}^{3}}\left[1+\frac{\bar{m}}{m_{s}}\right]+\frac{c}{3 \bar{m}^{2}}\left[1-2 \frac{\bar{m}}{m_{s}}\right], \\
\Sigma_{2}=a+\frac{c}{\bar{m}^{2}}=\Sigma_{2}^{*},
\end{gathered}
$$

TABLE III. Experimental mass splittings between light-quark baryons [6].

\begin{tabular}{ccc}
\hline \hline Splitting & Symbol & Value $(\mathrm{MeV})$ \\
\hline$M(p)-M(n)$ & $N_{1}$ & -1.2933 \\
$M\left(\Sigma^{+}\right)-M\left(\Sigma^{-}\right)$ & $\Sigma_{1}$ & $-8.08 \pm 0.08$ \\
$M\left(\Sigma^{+}\right)-2 M\left(\Sigma^{0}\right)+M\left(\Sigma^{-}\right)$ & $\Sigma_{2}$ & $1.535 \pm 0.090$ \\
$M\left(\Sigma^{*+}\right)-M\left(\Sigma^{*-}\right)$ & $\Sigma_{1}^{*}$ & $-4.40 \pm 0.61$ \\
$M\left(\Sigma^{*+}\right)-2 M\left(\Sigma^{* 0}\right)+M\left(\Sigma^{*-}\right)$ & $\Sigma_{2}^{*}$ & $2.6 \pm 2.1$ \\
$M\left(\Xi^{0}\right)-M\left(\Xi^{-}\right)$ & $\Xi_{1}$ & $-6.85 \pm 0.21$ \\
$M\left(\Xi^{* 0}\right)-M\left(\Xi^{*-}\right)$ & $\Xi_{1}^{*}$ & $-3.20 \pm 0.68$ \\
\hline \hline
\end{tabular}

TABLE IV. Predicted and observed isospin splittings in lightquark baryons.

\begin{tabular}{lrrrrrrr}
\hline \hline & $N_{1}$ & \multicolumn{1}{c}{$\Sigma_{1}$} & \multicolumn{1}{c}{$\Sigma_{1}^{*}$} & $\Sigma_{2}$ & $\Sigma_{2}^{*}$ & \multicolumn{1}{c}{$\Xi_{1}$} & \multicolumn{1}{c}{$\Xi_{1}^{*}$} \\
\hline Pred. & -1.293 & -8.087 & -4.685 & 1.529 & 1.529 & -6.794 & -3.392 \\
Expt. & -1.293 & -8.080 & -4.400 & 1.535 & 2.600 & -6.850 & -3.200 \\
Error $\sim 0$ & 0.080 & 0.610 & 0.090 & 2.100 & 0.210 & 0.680 \\
$\chi^{2}$ & 0.000 & 0.007 & 0.218 & 0.004 & 0.260 & 0.072 & 0.079 \\
\hline \hline & $\Xi_{1}=\Delta-\frac{2 a}{3}+\frac{4 b \Delta}{\bar{m}^{2} m_{s}}+\frac{4 c}{3 \bar{m} m_{s}}$,
\end{tabular}

The predicted isospin splittings are very close to the observed ones, since the Coleman-Glashow relation [16] $\Sigma_{1}=N_{1}+\Xi_{1}$ is very close to being obeyed by those quantities with the smallest experimental errors. The derived parameters are $\Delta=-2.49 \pm 0.04 \mathrm{MeV}, \quad a=$ $3.05 \pm 0.05 \mathrm{MeV}$, and $c / \bar{m}^{2}=-1.52 \pm 0.09 \mathrm{MeV}$. The predicted isospin splittings are compared with the observed ones in Table IV. The $\chi^{2}$ for the fit is 0.64 , driven mainly by the $\Sigma^{*}$ splittings.

In comparison with Ref. [2], the following relation is satisfied to greater accuracy:

$$
\begin{aligned}
\Sigma_{1} & -\Xi_{1}(=-1.23 \pm 0.22 \mathrm{MeV}) \\
& =\Sigma_{1}^{*}-\Xi_{1}^{*}(=-1.20 \pm 0.91 \mathrm{MeV}) .
\end{aligned}
$$

On the other hand, the relation

$\Sigma_{2}(=1.535 \pm 0.090 \mathrm{MeV})=\Sigma_{2}^{*}(=2.6 \pm 2.1 \mathrm{MeV})$

is still plagued with a large experimental error on the righthand side.

\section{Meson-baryon comparison}

The parameters $\Delta, a$, and $c / \bar{m}^{2}$ derived from fits to isospin splittings in mesons and baryons are compared in Table V. The signs are consistent, but central values are rather different. Slightly different parameters are obtained if one adopts a model in which quark masses are universal for mesons and baryons [4]. The difference between parameters obtained from mesons and baryons is not surprising in

TABLE V. Parameters describing isospin splittings in lightquark mesons and baryons.

\begin{tabular}{lccc}
\hline \hline & $\Delta$ & $a$ & $c / \bar{m}^{2}$ \\
\hline Meson & $-4.12 \pm 0.05$ & $2.20 \pm 0.62$ & $-2.32 \pm 0.21$ \\
Baryon & $-2.49 \pm 0.04$ & $3.05 \pm 0.05$ & $-1.52 \pm 0.09$ \\
\hline \hline
\end{tabular}


view of the large spread of values for $m_{u}-m_{d}$ obtained in various models (see Sec. IV in [2]).

\section{CHARMED HADRONS}

\section{A. Mesons}

The states at our disposal are summarized in Table VI. There is not enough information to derive a set of parameters describing these mass differences. However, the total spin-dependent terms contribute in a manner proportional to $\left\langle\sigma_{i} \cdot \sigma_{j}\right\rangle$, so one may write

$$
\begin{aligned}
& D_{1}=-\Delta_{c}^{m}+\frac{2 a_{c}^{m}}{3}-3 h_{c}^{m}, \\
& D_{1}^{*}=-\Delta_{c}^{m}+\frac{2 a_{c}^{m}}{3}+h_{c}^{m},
\end{aligned}
$$

where the superscript denotes charmed mesons. Eliminating the hyperfine contribution $h_{c}^{m}$, one finds

$$
-\Delta_{c}^{m}+\frac{2 a_{c}^{m}}{3}=3.76 \pm 0.05 \mathrm{MeV}
$$

This is to be compared with the corresponding value for light-quark mesons,

$$
\begin{aligned}
-\Delta^{m}+\frac{2 a^{m}}{3} & =[(4.12 \pm 0.05)+(1.47 \pm 0.41)] \mathrm{MeV} \\
& =(5.58 \pm 0.42) \mathrm{MeV}
\end{aligned}
$$

As for the hyperfine term $h_{c}^{m}=(-0.35 \pm 0.02) \mathrm{MeV}$, it contains both strong $b_{c}^{m}$ and electromagnetic $c_{c}^{m}$ contributions, which cannot be separated from one another without further assumptions.

\section{B. Baryons}

In analogy for the light-quark baryons, we write expressions for isospin splittings of charmed baryons:

$\Sigma_{c 1}=2 \Delta_{c}+\frac{5}{3} a_{c}+\frac{2 b_{c}}{\bar{m}^{2}} \frac{\Delta_{c}}{\bar{m}}\left[-1+2 \frac{\bar{m}}{m_{c}}\right]+\frac{c_{c}}{3 \bar{m}^{2}}\left[1-\frac{8 \bar{m}}{m_{c}}\right]$

TABLE VI. Masses and isospin splittings of charmed mesons, in $\mathrm{MeV}$.

\begin{tabular}{lc}
\hline \hline$D^{+}$ & $1869.65 \pm 0.05$ \\
$D^{0}$ & $1864.83 \pm 0.05$ \\
$D_{1} \equiv M\left(D^{+}\right)-M\left(D^{0}\right)$ & $4.822 \pm 0.015^{\mathrm{a}}$ \\
$D^{*+}$ & $2010.26 \pm 0.05$ \\
$D^{* 0}$ & $2006.85 \pm 0.05$ \\
$D_{1}^{*} \equiv M\left(D^{*+}\right)-M\left(D^{* 0}\right)$ & $3.41 \pm 0.07$ \\
\hline \hline
\end{tabular}

${ }^{\text {a } V a l u e ~ g i v e n ~ s e p a r a t e l y ~ i n ~ t h e ~} D^{0}$ section of [6].
$\Sigma_{c 1}^{*}=2 \Delta_{c}+\frac{5}{3} b_{c}-\frac{2 b_{c}}{\bar{m}^{2}} \frac{\Delta_{c}}{\bar{m}}\left[1+\frac{\bar{m}}{m_{c}}\right]+\frac{c_{c}}{3 \bar{m}^{2}}\left[1+\frac{4 \bar{m}}{m_{c}}\right]$

$$
\begin{gathered}
\Sigma_{c 2}=a_{c}+\frac{c_{c}}{\bar{m}^{2}}=\Sigma_{c 2}^{*} \\
\Xi_{c 1}=\Delta_{c}+\frac{1}{3} a_{c}+\frac{3 b_{c}}{\bar{m}^{2}} \frac{\Delta_{c}}{m_{s}}+\frac{c_{c}}{\bar{m} m_{s}} \\
\Xi_{c 1}^{\prime}=\Delta_{c}+\frac{1}{3} a_{c}+\frac{b_{c} \Delta_{c}}{\bar{m}^{2}}\left(\frac{2}{m_{c}}-\frac{1}{m_{s}}\right)-\frac{c_{c}}{3 \bar{m}}\left(\frac{1}{m_{s}}+\frac{4}{m_{c}}\right) \\
\Xi_{c 1}^{*}=\Delta_{c}+\frac{1}{3} a_{c}-\frac{b_{c} \Delta_{c}}{\bar{m}^{2}}\left(\frac{1}{m_{s}}+\frac{1}{m_{c}}\right)+\frac{c_{c}}{3 \bar{m}}\left(\frac{2}{m_{c}}-\frac{1}{m_{s}}\right) .
\end{gathered}
$$

We update a couple of relations, noted in Ref. [17], which follow from our assumptions. In 1998 the relation

\begin{tabular}{|c|c|}
\hline$M\left(\Sigma_{c}^{++}\right)$ & $2453.97 \pm 0.14$ \\
\hline$M\left(\Sigma_{c}^{+}\right)$ & $2452.9 \pm 0.4$ \\
\hline$M\left(\Sigma_{c}^{0}\right)$ & $2453.75 \pm 0.14$ \\
\hline$\Sigma_{c 1} \equiv M\left(\Sigma_{c}^{++}\right)-M\left(\Sigma_{c}^{0}\right)$ & $0.220 \pm 0.013^{\mathrm{a}}$ \\
\hline$\Sigma_{c 2} \equiv M\left(\Sigma_{c}^{++}\right)-2 M\left(\Sigma_{c}^{+}\right)+M\left(\Sigma_{c}^{0}\right)$ & $1.92 \pm 0.82$ \\
\hline$M\left(\Sigma_{c}^{*++}\right)$ & $2518.41_{-0.19}^{+0.21}$ \\
\hline$M\left(\Sigma_{c}^{*+}\right)$ & $2517.5 \pm 2.3$ \\
\hline$M\left(\Sigma_{c}^{* 0}\right)$ & $2518.48 \pm 0.20$ \\
\hline$\Sigma_{c 1}^{*} \equiv M\left(\Sigma_{c}^{*++}\right)-M\left(\Sigma_{c}^{* 0}\right)$ & $0.01 \pm 0.15^{\mathrm{b}}$ \\
\hline$\Sigma_{c 2}^{*} \equiv M\left(\Sigma_{c}^{++}\right)-2 M\left(\Sigma_{c}^{*+}\right)+M\left(\Sigma_{c}^{* 0}\right)$ & $1.89 \pm 4.61$ \\
\hline$M\left(\Xi_{c}^{+}\right)$ & $2467.93 \pm 0.18$ \\
\hline$M\left(\Xi_{c}^{0}\right)$ & $2470.91 \pm 0.25$ \\
\hline$\Xi_{c 1} \equiv M\left(\Xi_{c}^{+}\right)-M\left(\Xi_{c}^{0}\right)$ & $-2.98 \pm 0.22^{\mathrm{c}}$ \\
\hline$M\left(\Xi_{c}^{\prime+}\right)$ & $2578.4 \pm 0.5$ \\
\hline$M\left(\Xi_{c}^{\prime 0}\right)$ & $2579.2 \pm 0.5$ \\
\hline$\Xi_{c 1}^{\prime} \equiv M\left(\Xi_{c}^{\prime+}\right)-M\left(\Xi_{c}^{\prime 0}\right)$ & $-0.8 \pm 0.6^{\mathrm{d}}$ \\
\hline$M\left(\Xi_{c}^{*+}\right)$ & $2645.57 \pm 0.26$ \\
\hline$M\left(\Xi_{c}^{* 0}\right)$ & $2646.38 \pm 0.21$ \\
\hline$\Xi_{c 1}^{*} \equiv M\left(\Xi^{*+}\right)-M\left(\Xi^{* 0}\right)$ & $-0.80 \pm 0.26^{\mathrm{e}}$ \\
\hline $\begin{array}{l}{ }^{\mathrm{a}} \text { Listed in [6], } \Sigma_{c} \text { section. } \\
\text { b Listed in [6], } \Sigma_{c}^{*} \text { section. } \\
\text { L Listed in [6], } \Xi_{c} \text { section. } \\
{ }^{\mathrm{d}} \text { Listed in [6], } \Xi_{c}^{\prime+}(2578) \text { section. } \\
{ }^{\mathrm{d}} \text { Listed in [6], } \Xi_{c}(2645) \text { section. }\end{array}$ & \\
\hline
\end{tabular}

$$
\Sigma_{c 2} \equiv M\left(\Sigma_{c}^{++}\right)-2 M\left(\Sigma_{c}^{+}\right)+M\left(\Sigma_{c}^{0}\right)=\Sigma_{2}
$$

appeared to be violated [2], with the left-hand side giving $-2.0 \pm 1.3 \mathrm{MeV}$ while the right-hand side gave $1.71 \pm$ $0.18 \mathrm{MeV}$. The present status of charmed baryon masses and isospin splittings is summarized in Table VII. The

TABLE VII. Masses and isospin splittings of charmed baryons, in $\mathrm{MeV}$. 
TABLE VIII. Predicted and observed isospin splittings in charmed baryons. Errors are experimental values, used in calculating $\chi^{2}$ contributions.

\begin{tabular}{lccrcrrr}
\hline \hline & $\Sigma_{c 1}$ & $\Sigma_{c 2}$ & \multicolumn{1}{c}{$\Sigma_{c 1}^{*}$} & $\Sigma_{c 2}^{*}$ & \multicolumn{1}{c}{$\Xi_{c 1}$} & \multicolumn{1}{c}{$\Xi_{c 1}^{\prime}$} & \multicolumn{1}{c}{$\Xi_{c 1}^{*}$} \\
\hline Fit & 0.221 & 1.916 & -0.064 & 1.916 & -2.827 & -1.058 & -1.200 \\
Expt. & 0.220 & 1.920 & 0.010 & 1.890 & -2.980 & -0.800 & -0.800 \\
Error & 0.013 & 0.820 & 0.150 & 4.610 & 0.220 & 0.600 & 0.260 \\
$\chi^{2}$ & 0.003 & 0.000 & 0.243 & 0.000 & 0.484 & 0.185 & 2.369 \\
\hline \hline
\end{tabular}

sum rule is now satisfied, with the left-hand side giving $1.92 \pm 0.82 \mathrm{MeV}$ while the right-hand side gives $1.535 \pm$ $0.090 \mathrm{MeV}$.

Another sum rule [17],

$$
\Sigma_{c 1}-2 \Xi_{c 1}^{\prime}=\Sigma_{1}^{*}-2 \Xi_{1}^{*},
$$

is beginning to be tested, with the left-hand side yielding $1.8 \pm 1.2 \mathrm{MeV}$ while the right-hand side is $2.0 \pm$ $1.5 \mathrm{MeV}$. The large errors are associated both with $\Xi_{c 1}^{\prime}$ and $\Xi_{1}^{*}$. A further relation is

$$
\Sigma_{c 1}^{*}-2 \Xi_{c 1}^{*}=\Sigma_{1}^{*}-2 \Xi_{1}^{*},
$$

where the left-hand side is $1.61 \pm 0.54 \mathrm{MeV}$. The sum rule is satisfied, with the main uncertainty coming from the right-hand side.

The information about charmed baryons is complete enough that one can perform a fit to their isospin splittings, determining parameters $\Delta_{c}, a_{c}$, and $c_{c} / \bar{m}^{2}$ which may be compared with their light-quark counterparts. Fixed parameters in this fit (see the caption to Table I, with $m_{c}$ taken from [12]) are

$$
\begin{aligned}
\bar{m} & =362.1 \mathrm{MeV}, \quad m_{s}=543.9 \mathrm{MeV}, \\
b_{c} /\left(\bar{m}^{2}\right) & =b /(\bar{m})^{2}=50.0 \mathrm{MeV}, \quad m_{c}=1710.5 \mathrm{MeV} .
\end{aligned}
$$

The results of this fit are summarized in Table VIII. The derived parameters are $\Delta_{c}=-2.49 \pm 0.20 \mathrm{MeV}, a_{c}=$ $2.77 \pm 0.23 \mathrm{MeV}$, and $c_{c} / \bar{m}^{2}=-0.85 \pm 0.15 \mathrm{MeV}$. The first two are rather close to those obtained for light-quark baryons, while the last is of the same sign but only about half as large as $c / \bar{m}^{2}$. The $\chi^{2}$ for the fit is 3.28 , driven mainly by $\Xi_{c 1}^{*}$.

\section{BEAUTY HADRONS}

\section{A. Mesons}

The information on beauty mesons relevant for analysis of isospin splittings is summarized in Table IX. An analysis parallel to that for charmed mesons is not possible in the absence of a value of $M\left(B^{* 0}\right)$. Thus in analogy to Eq. (17) all we can write is
TABLE IX. Masses and isospin splittings of beauty mesons, in $\mathrm{MeV}$.

\begin{tabular}{cr}
\hline \hline$B^{+}$ & $5279.33 \pm 0.13$ \\
$B^{0}$ & $5279.64 \pm 0.14$ \\
$B_{1} \equiv M\left(B^{+}\right)-M\left(B^{0}\right)$ & $-0.31 \pm 0.07$ \\
$M\left(B^{*+}\right)-M\left(B^{+}\right)$ & $45.37 \pm 0.21$ \\
$M\left(B^{*+}\right)$ & $5324.70 \pm 0.27$ \\
$M\left(B^{* 0}\right)$ & $\cdots$ \\
$B_{1}^{*} \equiv M\left(B^{*+}\right)-M\left(B^{* 0}\right)$ & $\cdots$ \\
\hline \hline
\end{tabular}

$$
\begin{aligned}
& B_{1}=\Delta_{b}^{m}+\frac{a_{b}^{m}}{3}-3 h_{b}^{m}, \\
& B_{1}^{*}=\Delta_{b}^{m}+\frac{a_{b}^{m}}{3}+h_{b}^{m} .
\end{aligned}
$$

Eliminating the spin-dependent term $h_{b}^{m}$, one finds

$$
\Delta_{b}^{m}+\frac{a_{b}^{m}}{3}=\frac{1}{4}\left(B_{1}+3 B_{1}^{*}\right) .
$$

Now, $h_{b}^{m}$ contains quark charges different from those in $h_{c}^{m}$, but is smaller in magnitude by about a factor of $m_{b}^{m} / m_{c}^{m} \simeq 3$. Thus we probably make an error of only about $0.1 \mathrm{MeV}$ in neglecting it. In that case we would predict $B_{1}^{*} \simeq B_{1} \simeq-0.31 \pm 0.07 \mathrm{MeV}$. This is consistent with the Particle Data Group's charge-averaged value $M\left(B^{*}\right)-$ $M(B)=45.22 \pm 0.21 \mathrm{MeV}$, to be compared with $M\left(B^{*+}\right)-$ $M\left(B^{+}\right)=45.37 \pm 0.21 \mathrm{MeV}$ [6], implying that $B^{*}$ and $B$ isospin splittings are not too different from one another. Definitive conclusions await the measurement of $M\left(B^{* 0}\right)$. For light-quark mesons, the combination $\Delta^{m}+\frac{a^{m}}{3}$ is equal to $(-4.12+0.71) \mathrm{MeV}=-3.41 \mathrm{MeV}$.

\section{B. Baryons}

The relevant masses of beauty baryons are summarized in Table X. Here we only have information on $\Delta I=1$

TABLE X. Masses and isospin splittings of beauty baryons, in $\mathrm{MeV}$.

\begin{tabular}{cc}
\hline \hline$M\left(\Sigma_{b}^{+}\right)$ & $5810.56 \pm 0.25$ \\
$M\left(\Sigma_{b}^{-}\right)$ & $5815.64 \pm 0.27$ \\
$\Sigma_{b 1} \equiv M\left(\Sigma_{b}^{+}\right)-M\left(\Sigma_{b}^{-}\right)$ & $-5.06 \pm 0.18^{\mathrm{a}}$ \\
$M\left(\Sigma_{b}^{*+}\right)$ & $5830.32 \pm 0.27$ \\
$M\left(\Sigma_{b}^{*-}\right)$ & $5834.74 \pm 0.30$ \\
$\Sigma_{b 1}^{*} \equiv M\left(\Sigma_{b}^{*+}\right)-M\left(\Sigma_{b}^{*-}\right)$ & $-4.37 \pm 0.33^{\mathrm{a}}$ \\
$M\left(\Xi_{b}^{0}\right)$ & $5791.8 \pm 0.5^{\mathrm{b}}$ \\
$M\left(\Xi_{b}^{-}\right)$ & $5797.0 \pm 0.9^{\mathrm{c}}$ \\
$\Xi_{b 1} \equiv M\left(\Xi_{b}^{0}\right)-M\left(\Xi_{b}^{-}\right)$ & $-5.9 \pm 0.6$ \\
$M\left(\Xi_{b}^{* 0}\right)$ & $5952.3 \pm 0.9$ \\
$M\left(\Xi_{b}^{*-}\right)$ & $5955.33 \pm 0.13$ \\
$\Xi_{b 1}^{*} \equiv M\left(\Xi_{b}^{* 0}\right)-M\left(\Xi_{b}^{*-}\right)$ & $-3.03 \pm 0.91$ \\
\hline \hline
\end{tabular}

${ }^{a}$ From PDGLive [6].

${ }^{\mathrm{b}} \mathrm{LHCb}$ value [18].

${ }^{c} \mathrm{LHCb}$ value [19]. 
splittings, as the neutral $\Sigma_{b}$ and $\Sigma_{b}^{*}$ masses are still unmeasured. The decomposition of isospin splittings in terms of $\Delta_{b}, a_{b}, b_{b}$, and $c_{b}$ is

$$
\Sigma_{b 1}=2 \Delta_{b}-\frac{1}{3} a_{b}-\frac{2 b_{b} \Delta_{b}}{\bar{m}^{3}}\left[1-\frac{2 \bar{m}}{m_{b}}\right]+\frac{c_{b}}{3 \bar{m}^{2}}\left[1+\frac{4 \bar{m}}{m_{b}}\right],
$$

$$
\Sigma_{b 1}^{*}=2 \Delta_{b}-\frac{1}{3} a_{b}-\frac{2 b_{b} \Delta_{b}}{\bar{m}^{3}}\left[1+\frac{\bar{m}}{m_{b}}\right]+\frac{c_{b}}{3 \bar{m}^{2}}\left[1-2 \frac{\bar{m}}{m_{b}}\right]
$$

$$
\begin{gathered}
\Sigma_{b 2}=a_{b}+\frac{c_{b}}{\bar{m}^{2}}=\Sigma_{b 2}^{*}, \\
\Xi_{b 1}=\Delta_{b}-\frac{2}{3} a_{b}+\frac{3 b_{b} \Delta_{b}}{\bar{m}^{2} m_{s}}+\frac{c_{b}}{\bar{m} m_{s}}, \\
\Xi_{b 1}^{\prime}=\Delta_{b}-\frac{2}{3} a_{b}+\frac{\Delta_{b} b_{b}}{\bar{m}^{2}}\left[\frac{2}{m_{b}}-\frac{1}{m_{s}}\right]+\frac{c_{b}}{3 \bar{m}}\left[\frac{2}{m_{b}}-\frac{1}{m_{s}}\right]
\end{gathered}
$$

$\Xi_{b 1}^{*}=\Delta_{b}-\frac{2}{3} a_{b}-\frac{\Delta_{b} b_{b}}{\bar{m}^{2}}\left[\frac{1}{m_{s}}+\frac{1}{m_{b}}\right]-\frac{c_{b}}{3 \bar{m}}\left[\frac{1}{m_{s}}+\frac{1}{m_{b}}\right]$

One may perform a fit to these quantities, varying $\Delta_{b}, a_{b}$, and $c_{b} / \bar{m}^{2}$. Fixed parameters in this fit (see the caption of Table I, with $m_{b}$ taken from [12]) are

$$
\begin{aligned}
\bar{m} & =362.1 \mathrm{MeV}, \quad m_{s}=543.9 \mathrm{MeV}, \\
b_{b} /\left(\bar{m}^{2}\right) & =b /(\bar{m})^{2}=50.0 \mathrm{MeV}, \quad m_{b}=5043.5 \mathrm{MeV} .
\end{aligned}
$$

The results are shown in Table XI. The associated $\chi^{2}$ is 0.33 , so a consistent set of parameters is obtained. However, they differ from those fitting the light-quark or charmed baryons: in $\mathrm{MeV}$,

TABLE XI. Predicted and observed isospin splittings in beauty baryons. Errors are experimental values, used in calculating $\chi^{2}$ contributions.

\begin{tabular}{lrrrrrrr}
\hline \hline & $\Sigma_{b 1}$ & $\Sigma_{b 2}$ & $\Sigma_{b 1}^{*}$ & $\Sigma_{b 2}^{*}$ & $\Xi_{b 1}$ & $\Xi_{b 1}^{\prime}$ & $\Xi_{b 1}^{*}$ \\
\hline Fit & -5.015 & 0.410 & -4.522 & 0.410 & -5.979 & -3.095 & -2.848 \\
Expt. & -5.060 & $\cdots$ & -4.370 & $\cdots$ & -5.900 & $\cdots$ & 3.030 \\
Error & 0.180 & $\cdots$ & 0.330 & $\cdots$ & 0.600 & $\cdots$ & 0.910 \\
$\chi^{2}$ & 0.063 & $\cdots$ & 0.211 & $\cdots$ & 0.017 & $\cdots$ & 0.040 \\
\hline \hline
\end{tabular}

$$
\begin{aligned}
\Delta_{b} & =-1.56 \pm 0.34, \quad a_{b}=3.20 \pm 1.17, \\
c_{b} / \bar{m}^{2} & =-2.79 \pm 1.18 .
\end{aligned}
$$

Two relations analogous to those for charmed baryons are predicted:

$$
\Sigma_{b 1}-2 \Xi_{b 1}^{\prime}=\Sigma_{b 1}^{*}-2 \Xi_{b 1}^{*}=\Sigma_{1}^{*}-2 \Xi_{1}^{*},
$$

with the second holding only for equal light-quark baryon and beauty baryon parameters. The right-hand side of this relation is

$$
\text { rhs }=a-\frac{2 \Delta b}{\bar{m}^{2}}\left[\frac{1}{\bar{m}}-\frac{1}{m_{s}}\right]+\frac{c}{3 \bar{m}^{2}}\left[1+\frac{2 \bar{m}}{m_{s}}\right],
$$

whether for light-quark, charmed, or beauty baryons. In Sec. IV we found $\Sigma_{1}^{*}-2 \Xi_{1}^{*}=2.0 \pm 1.5 \mathrm{MeV}$. However, the large splitting between neutral and charged $\Xi_{b}$ states leads the middle term of this sum rule to the value

$$
\begin{aligned}
\Sigma_{b 1}^{*}-2 \Xi_{b 1}^{*} & =[-4.37 \pm 0.33+2(5.9 \pm 0.6)] \mathrm{MeV} \\
& =(7.4 \pm 1.2) \mathrm{MeV}
\end{aligned}
$$

The violation of this sum rule is further evidence that one cannot always assume equal values of $\Delta, a, c$ for bottomand lighter-quark systems.

\section{CHARM-BEAUTY RELATIONS}

\section{A. Universal parameters?}

The comparison of isospin-violating parameters among light-quark, charmed, and beauty hadrons shows that one cannot regard them as universal. Suppose, first of all, that one took $\Delta^{m}=\Delta_{c}^{m}=\Delta_{b}^{m}$. With this assumption one could solve Eqs. (19) and (31) to obtain $a_{c}^{m}=-0.54 \mathrm{MeV}$ and $a_{b}^{m}=11.42 \mathrm{MeV}$. This makes little sense because the parameter $a_{c}^{m}$ should be positive.

One could, instead, assume that the heavy-quark parameters

$$
\Delta_{Q}^{m} \equiv \Delta_{c}^{m}=\Delta_{b}^{m}, \quad a_{Q}^{m} \equiv a_{c}^{m}=a_{b}^{m}
$$

are equal for charmed and beauty mesons. (As we shall see, this is approximately true for baryons.) Then solving Eqs. (19) and (33), assuming $h_{b}^{m}=0$, one finds

$$
\Delta_{Q}^{m}=-1.46 \pm 0.05 \mathrm{MeV}, \quad a_{Q}^{m}=3.45 \pm 0.09 \mathrm{MeV}
$$

to be compared with the light-quark meson value (see Sec. III A) 
TABLE XII. Masses (in MeV) contributing to relation (48) between charmed and beauty meson hyperfine splittings.

\begin{tabular}{cc}
\hline \hline State & Mass \\
\hline $\bar{B}_{s}^{*}$ & $5415.4_{-1.5}^{+1.8}$ \\
$\bar{B}_{s}$ & $5366.88 \pm 0.17$ \\
$\bar{B}_{s}^{*}-\bar{B}_{s}$ & $48.6_{-1.5}^{+1.8}$ \\
$\bar{B}^{* 0}$ & $5324.70 \pm 0.22^{\mathrm{a}}$ \\
$\bar{B}^{0}$ & $5279.63 \pm 0.15$ \\
$\bar{B}^{* 0}-\bar{B}^{0}$ & $45.07 \pm 0.21^{\mathrm{b}}$ \\
$D_{s}^{*}$ & $2112.2 \pm 0.4$ \\
$D_{s}$ & $1968.34 \pm 0.07$ \\
$D_{s}^{*}-D_{s}$ & $143.86 \pm 0.41$ \\
$D^{*+}$ & $2010.26 \pm 0.05$ \\
$D^{+}$ & $1869.65 \pm 0.05$ \\
$D^{*+}-D^{+}$ & $140.603 \pm 0.015$ \\
\hline \hline
\end{tabular}

${ }^{\text {a}}$ The charge of the state is not specified in Ref. [6]. Instead, we quote the value for a production-weighted average.

${ }^{b}$ Estimate based on small isospin splitting between charged and neutral $\bar{B}^{*}$.

$\Delta^{m}=-4.12 \pm 0.05 \mathrm{MeV}, \quad a^{m}=2.20 \pm 0.62 \mathrm{MeV}$

The larger value of $a$ makes sense, because of the deeper binding of charmed and bottom hadrons (hence a larger expectation value of $1 / r$ ). However, the difference between
$\Delta_{Q}^{m}$ [close to the value in Eq. (41)] and $\Delta^{m}$ is somewhat puzzling. Note that in Table $\mathrm{V}$ we found $\Delta=$ $-2.49 \pm 0.04 \mathrm{MeV}$ for light-quark baryons, considerably different from the value $\Delta^{m}$.

\section{B. Relations between hyperfine splittings}

Although it is not an isospin splitting, a relation between charmed meson and beauty meson hyperfine splittings makes use of the relatively new result from the $B A B A R$ Collaboration [5] which enters the Particle Data Group compilation. The relation [1] (updated in Ref. [7] to account for QCD corrections) is

$$
\begin{aligned}
& M\left(\bar{B}_{s}^{*}\right)-M\left(\bar{B}_{s}\right)-\left[M\left(\bar{B}^{* 0}\right)-M\left(\bar{B}^{0}\right)\right] \\
& \quad=\left(m_{c} / m_{b}\right)\left\{M\left(D_{s}^{*}\right)-M\left(D_{s}\right)-\left[M\left(D^{*+}-M\left(D^{+}\right)\right]\right\} .\right.
\end{aligned}
$$

The left- and right-hand sides of this equation, based on heavy-quark symmetry, are related to one another by $b \leftrightarrow c$. The present status of its terms is summarized in Table XII [6]. The left-hand side of Eq. (48) is $3.5 \pm 1.7 \mathrm{MeV}$, while the right-hand side is $\left(m_{c} / m_{b}\right)(3.26 \pm 0.41) \mathrm{MeV} \simeq$ $(1.09 \pm 0.14) \mathrm{MeV}$. A decisive test of this relation awaits separate measurements of the masses of $\bar{B}^{*+}$ and $\bar{B}^{* 0}$ and a reduced error on the mass of $B^{*} s$.

\begin{tabular}{|c|c|c|c|}
\hline Reference & $\Delta$ or $m_{u}-m_{d}(\mathrm{MeV})$ & $a(\mathrm{MeV})$ & Comments \\
\hline This work & $\begin{array}{l}\Delta^{m}=-4.117 \\
\Delta^{b}=-2.491\end{array}$ & $\begin{array}{l}a^{m}=2.119 \\
a^{b}=3.052\end{array}$ & $\begin{array}{l}\text { Light-quark meson octet } \\
\text { Light-quark baryons }\end{array}$ \\
\hline [2] & $\Delta^{b}=-2.57^{\mathrm{a}}$ & $a^{b}=3.06^{\mathrm{a}}$ & Neglecting kinetic term $K$ \\
\hline [3] & & $a^{m}=3.18 \pm 0.48$ & Eq. (15) and Appendix A \\
\hline [4] & $\begin{array}{l}\Delta^{b}=-2.48^{\mathrm{a}} \\
\Delta^{b}=-2.67^{\mathrm{b}}\end{array}$ & $\begin{array}{l}a^{b}=3.05^{\mathrm{a}} \\
a^{b}=2.83^{\mathrm{b}}\end{array}$ & \\
\hline [6] & $m_{u}-m_{d}=-2.55 \pm 0.25$ & & $\overline{\mathrm{MS}}, \mu_{\text {renorm. }}=2 \mathrm{GeV}$ \\
\hline [15] & $m_{u}-m_{d}=-6$ & & \\
\hline [24] & $m_{u}-m_{d}=-3.8$ & & \\
\hline [25] & $m_{u}-m_{d}=-2.54 \pm 0.04$ & & J. Franklin, priv. commun. \\
\hline [26] & $m_{u}-m_{d}=-2.66$ & $a^{m}=1.5 \pm 0.5$ & Baryon $a$ unclear \\
\hline [27] & $m_{u}-m_{d}=-4.12$ & & MIT bag model \\
\hline [28] & $m_{u}-m_{d}=-6.7$ & & MIT bag model \\
\hline [29] & $m_{u}-m_{d}=-4.4$ & $a^{b}=2.9$ & \\
\hline [30] & $m_{u}-m_{d}=-2.4$ & Ignored & "Photon cloud" effects \\
\hline [33] & $m_{u}-m_{d}=-11$ & 3.39 & Potential models \\
\hline [35] & $m_{u}-m_{d}=-1.88$ & 3.52 & Including three-body terms \\
\hline [36] & $m_{u}-m_{d}=-1.82$ & & \\
\hline [38] & $\Delta^{b}=-1.84 \pm 0.16$ & & \\
\hline [39] & $m_{u}-m_{d}=-2.5$ & & \\
\hline [42] & $m_{u}-m_{d}=-5.7$ & & $\overline{\mathrm{MS}}, \mu_{\text {renorm. }}=100 \mathrm{MeV}$ \\
\hline & $m_{u}-m_{d}=-4.7$ & & $\overline{\mathrm{MS}}, \mu_{\mathrm{renorm}}=200 \mathrm{MeV}$ \\
\hline
\end{tabular}

TABLE XIII. Comparison of parameters governing isospin splittings in quark models.

${ }^{\mathrm{a}}$ Different masses for quarks in mesons and baryons.

${ }^{\mathrm{b}}$ Universal masses for quarks in mesons and baryons. 
TABLE XIV. Observables needed to refine understanding of isospin breaking.

\begin{tabular}{cc}
\hline \hline Observable & Value, if known \\
\hline$M\left(B^{* 0}\right)$ & $\ldots$ \\
$M\left(K^{* \pm}\right)$ & $891.76 \pm 0.25 \mathrm{MeV}$ \\
& hadroproduction \\
& $895.5 \pm 0.8 \mathrm{MeV}$, \\
& $\tau$ decay \\
$M\left(B_{s}^{*}\right)$ & $5415.4_{-1.5}^{+1.8} \mathrm{MeV}$ \\
$M\left(\Sigma^{*+}\right)-2 M\left(\Sigma^{* 0}\right)+M\left(\Sigma^{*-}\right)$ & $2.6 \pm 2.1 \mathrm{MeV}$ \\
$M\left(\Xi_{c c}^{++}\right)-M\left(\Xi_{c c}^{+}\right)$ & Predicted in Ref. [4] \\
\hline \hline
\end{tabular}

\section{COMPARISON WITH OTHER APPROACHES}

Thanks to improvements in computing power, lattice quantum chromodynamics (LQCD) is beginning to be able to take into account isospin splittings in masses and decay constants. (For some references on the latter, see [20].) For LQCD approaches to light-quark splittings see Refs. [21,22] (octet baryons) and [23] (octet mesons and baryons). We look forward to LQCD calculations of isospin splittings in mesons and baryons containing at least one heavy quark.

Within quark models there is a long history of tackling isospin splittings in hadrons [2-4,15,24-41]. (Reference [40], though using chiral perturbation theory, gives an extensive list of works based on quark models.) The parameters $\Delta$ (or $\left.m_{u}-m_{d}\right)$ and $a$, when given, are compared in Table XIII. We show there also the latest estimate of $m_{u}-m_{d}$ in the current-quark picture [6].

The relation between current-quark masses (see the minireview No. 66 in Ref. [6], and the formalism set forth in Ref. [42]) and the constituent-quark masses we are using has been discussed in [15]. However, it has been pointed out in [6] that this relation (and hence the definition of constituent-quark masses) is model dependent. We note that many of our determinations of $m_{u}-m_{d}$ in the constituent-quark picture are not that far from the current-quark value of $\sim-2.5 \mathrm{MeV}$, , suggesting that in those cases the QCD "dressing" of current quarks may act linearly on their masses. (An exception is presented by

\footnotetext{
${ }^{1}$ At a scale of $2 \mathrm{GeV}$, one recent lattice QCD determination [43] finds $m_{u}=2.130(41) \mathrm{MeV}, m_{d}=4.675(56) \mathrm{MeV}$, while another [44] finds $m_{u}=2.50 \pm 0.17 \mathrm{MeV}, m_{d}=4.88 \pm 0.20 \mathrm{MeV}$.
}

the light-quark mesons, for which $\left|m_{u}-m_{d}\right|$ is considerably larger, and by the strange-quark mass, which is about $90 \mathrm{MeV}$ heavier than the average nonstrange mass in the current-quark picture $[6,43]$ but $180 \mathrm{MeV}$ heavier than the average nonstrange mass in our constituent-quark picture (see the caption of Table I).

\section{CONCLUSIONS}

Within a constituent-quark picture, we have updated predictions of isospin splittings in hadrons with at most one $c$ or $b$ quark. Effects considered included an intrinsic $u-d$ mass difference and its effect on kinetic energies (parameter $\Delta)$; Coulomb interactions among the constituent quarks (parameter $a$ ); and quark mass dependence on strong and electromagnetic hyperfine splittings (parameters $b$ and $c$, respectively). The parameter $\Delta$ is found to have a nonuniversal value, ranging from $-4.1 \mathrm{MeV}$ in light-quark mesons to $-1.5 \mathrm{MeV}$ in heavy-quark mesons and possibly in $b$-quark baryons. This latter conclusion is preliminary in the absence of a direct measurement of the masses of both $B^{*}$ charge states. A value of $\Delta$ near $-2.5 \mathrm{MeV}$ seems consistent with isospin splittings in light-quark and charmed baryons, but more negative than in bottom baryons. Most estimates of the Coulomb interaction term $a$ lie between 2 and $3 \mathrm{MeV}$.

Quantities whose measurement would help to test relations in the present analysis include improved masses of $K^{* \pm}$ and $B_{s}^{*}$; some isospin splittings in beauty baryons; and $M\left(\Xi_{c c}^{++}\right)-M\left(\Xi_{c c}^{+}\right)$, predicted in Ref. [4] to be (2.17 \pm $0.11) \mathrm{MeV}$ under the present set of assumptions [or $(1.49 \pm 0.12) \mathrm{MeV}$ in a model with universal quark masses for mesons and baryons.] We look forward to these developments, summarized in Table XIV, in the data. Our survey of isospin-violating effects has shown that a description in terms of universal $u-d$ mass differences and effective Coulomb and hyperfine interactions has its limitations, with effective mass splittings dependent to some degree on the hadronic environment.

\section{ACKNOWLEDGMENTS}

We thank Tom DeGrand for a question leading to the present investigation; Mike Sokoloff for alerting us to Refs. [5,7] and for a useful conversation; and Davide Giusti and Feng-Kun Guo for helpful comments on the manuscript. 
[1] J. L. Rosner and M. B. Wise, Meson masses from SU(3) and heavy quark symmetry, Phys. Rev. D 47, 343 (1993).

[2] J. L. Rosner, Improved tests of relations for baryon isomultiplet splittings, Phys. Rev. D 57, 4310 (1998).

[3] S. J. Brodsky, F. K. Guo, C. Hanhart, and U. G. Meissner, Isospin splittings of doubly heavy baryons, Phys. Lett. B 698, 251 (2011).

[4] M. Karliner and J. L. Rosner, Isospin splittings in baryons with two heavy quarks, Phys. Rev. D 96, 033004 (2017).

[5] J. P. Lees et al..(BABAR Collaboration), Measurement of the $D^{*+}(2010)-D^{+}$Mass Difference, Phys. Rev. Lett. 119, 202003 (2017).

[6] M. Tanabashi et al. (Particle Data Group), Review of particle physics, Phys. Rev. D 98, 030001 (2018).

[7] J. L. Goity and C. P. Jayalath, Strong and electromagnetic mass splittings in heavy mesons, Phys. Lett. B 650, 22 (2007).

[8] V. Fanti et al. (NA48 Collaboration), Precision measurement of the $\Xi^{0}$ mass and the branching ratios of the decays $\Xi^{0} \rightarrow \Lambda \gamma$ and $\Xi^{0} \rightarrow \Sigma^{0} \gamma$, Eur. Phys. J. C 12, 69 (2000).

[9] A. De Rújula, H. Georgi, and S. L. Glashow, Hadron masses in a gauge theory, Phys. Rev. D 12, 147 (1975).

[10] H. J. Lipkin, A unified description of mesons, baryons, and baryonium, Phys. Lett. 74B, 399 (1978).

[11] S. Gasiorowicz and J. L. Rosner, Hadron spectra and quarks, Am. J. Phys. 49, 954 (1981).

[12] M. Karliner and J. L. Rosner, Baryons with two heavy quarks: Masses, production, decays, and detection, Phys. Rev. D 90, 094007 (2014).

[13] R. Aaij et al. (LHCb Collaboration), Observation of the Doubly Charmed Baryon $\Xi_{c c}^{++}$, Phys. Rev. Lett. 119, 112001 (2017).

[14] M. Karliner, S. Nussinov, and J. L. Rosner, $Q Q \bar{Q} \bar{Q}$ states: Masses, production, and decays, Phys. Rev. D 95, 034011 (2017).

[15] N. Isgur, Isospin violating mass differences and mixing angles: The role of quark masses, Phys. Rev. D 21, 779 (1980); Erratum, Phys. Rev. D 23, 817(E) (1981).

[16] S. Coleman and S. L. Glashow, Departures from the eightfold way: Theory of strong interaction symmetry breakdown, Phys. Rev. 134, B671 (1964).

[17] J. Franklin, Mass relations for charmed baryons, Phys. Rev. D 12, 2077 (1975).

[18] R. Aaij et al. (LHCb Collaboration), Precision Measurement of the Mass and Lifetime of the $\Xi_{b}^{0}$ Baryon, Phys. Rev. Lett. 113, 032001 (2014).

[19] R. Aaij et al. (LHCb Collaboration), Precision Measurement of the Mass and Lifetime of the $\Xi_{b}^{-}$Baryon, Phys. Rev. Lett. 113, 242002 (2014).

[20] J. L. Rosner, S. L. Stone, and R. S. Van de Water, Minireview of leptonic decays of charged pseudoscalar mesons, in [6], article No. 84, p. 700, http://pdg.lbl.gov/2019/ reviews/rpp2018-rev-pseudoscalar-meson-decay-cons.pdf.

[21] S. Borsanyi et al. (Budapest-Marseille-Wuppertal Collaboration), Isospin Splittings in the Light Baryon Octet from Lattice QCD and QED, Phys. Rev. Lett. 111, 252001 (2013).

[22] S. Borsanyi et al., Ab initio calculation of the neutronproton mass difference, Science 347, 1452 (2015).

[23] R. Horsley et al., Isospin splittings of meson and baryon masses from three-flavor lattice QCD + QED, J. Phys. G 43, 10LT02 (2016).
[24] C. Itoh, T. Minamikawa, K. Miura, and T. Watanabe, Electromagnetic mass differences of lowest lying hadrons and behavior of quarks in hadrons, Prog. Theor. Phys. 61, 548 (1979).

[25] J. Franklin and D. B. Lichtenberg, Estimate of the quarkgluon coupling strength from baryon masses, Phys. Rev. D 25, 1997 (1982).

[26] L. H. Chan, Isospin mass splittings of hadrons with heavy quarks, Phys. Rev. D 31, 204 (1985).

[27] K. P. Tiwari, C. P. Singh, and M. P. Khanna, Electromagnetic mass splittings of heavier hadrons in the MIT bag model, Phys. Rev. D 31, 642 (1985).

[28] W. Y. P. Hwang and D. B. Lichtenberg, Mass splitting of heavy baryon isospin multiplets, Phys. Rev. D 35, 3526 (1987).

[29] S. Capstick, Isospin violations in baryons, and the $\Sigma_{c}^{0}-\Sigma_{c}^{++}$ mass difference, Phys. Rev. D 36, 2800 (1987).

[30] R. C. Verma and S. Srivastava, Photon cloud effects on isomultiplet mass differences of charmed and uncharmed baryons, Phys. Rev. D 38, 1623 (1988).

[31] R. E. Cutkosky and P. Geiger, Isospin splitting in heavy baryons and mesons, Phys. Rev. D 48, 1315 (1993).

[32] J. Franklin, Sum rules for charmed baryon masses, Phys. Rev. D 53, 564 (1996).

[33] M. Genovese, J. M. Richard, B. Silvestre-Brac, and K. Varga, Isospin mass splittings of baryons in potential models, Phys. Rev. D 59, 014012 (1999).

[34] B. Silvestre-Brac, F. Brau, and C. Semay, Electromagnetic splitting for mesons and baryons using dressed constituent quarks, J. Phys. G 29, 2685 (2003).

[35] L. Durand and P. Ha, Electromagnetic corrections to baryon masses, Phys. Rev. D 71, 073015 (2005); Erratum, Phys. Rev. D 76, 039903(E) (2007).

[36] P. Ha, Estimates of isospin breaking contributions to baryon masses, Phys. Rev. D 76, 073004 (2007).

[37] P. Ha, A parametrization of the baryon octet, and decuplet masses, J. Phys. G 35, 075006 (2008).

[38] C. W. Hwang and C. H. Chung, Isospin mass splittings of heavy baryons in HQS, Phys. Rev. D 78, 073013 (2008).

[39] H. Fritzsch, Isospin mass differences of heavy baryons, arXiv:0811.0481.

[40] F. K. Guo, C. Hanhart, and U. G. Meissner, Mass splittings within heavy baryon isospin multiplets in chiral perturbation theory, J. High Energy Phys. 09 (2008) 136.

[41] G. Dillon and G. Morpurgo, The general QCD parametrization and the hierarchy of its parameters: Why some simple models of hadrons work so well, Riv. Nuovo Cimento 33, 1 (2010).

[42] J. Gasser and H. Leutwyler, Quark masses, Phys. Rep. 87, 77 (1982).

[43] A. Bazavov et al. (Fermilab Lattice, MILC, and TUMQCD Collaborations), Up-, down-, strange-, charm-, and bottomquark masses from four-flavor lattice QCD, Phys. Rev. D 98, 054517 (2018).

[44] D. Giusti, V. Lubicz, C. Tarantino, G. Martinelli, S. Sanfilippo, S. Simula, and N. Tantalo, Leading isospinbreaking corrections to pion, kaon and charmed-meson masses with twisted-mass fermions, Phys. Rev. D 95, 114504 (2017). 